\title{
Midazolam en la prevención del espasmo de la arteria radial. Ensayo clínico aleatorizado a doble ciegas
}

\author{
Midazolam in the prevention of spasm of the radial artery. Randomized \\ double blind clinical trial
}

Suilbert Rodríguez Blanco', Abel Leyva Quert', José Luis Mendoza Ortiz', Ángela Rosa Gutiérrez Rojas². Giovanny Ponte González', José M. Aguilar Medina', Juan C. Pérez Guerra', Daysi Luperon Laforte'

\section{RESUMEN}

Introducción. El acceso radial en el intervencionismo coronario percutáneo ha emergido como la vía preferida por muchos cardiólogos intervencionistas, dada la evidencia que la asocia a menores complicaciones, menos estadía hospitalaria y mejores resultados.Su principal problema es el espasmo de la arteria radial, complicación que puede llevar a la necesidad de cambiar el sitio de acceso arterial, incrementando la duración del proceder, su complejidad y las complicaciones vasculares. Objetivo. Evaluar la eficacia y seguridad del midazolam en la prevención del espasmo radial en el cateterismo cardíaco por vía transradial. Material y Métodos. Ensayo clínico controlado, aleatorizado y a doble ciegas. Donde se aleatorizaron 300 pacientes ( 2 grupos de 150), al grupo de tratamiento se le administró midazolam previo al proceder y al grupo control solución salina. Resultados. Se documentó espasmo radial en 102 pacientes (34\%). En el grupo de midazolam se logró una reducción de la incidencia de espasmo:19 $(18,6 \%)$ vs $83(81,4 \%) ; p=0.0004$; RR: 0,28 $(0,49-0,43)$. Se redujo significativamente la necesidad de cambio de vía de acceso arterial, $p=0,0049$; RR: $0,61(0,17-1,01)$. Solo se documentaron 3 pacientes con somnolencia como evento adverso al fármaco. Conclusiones. El uso de Midazolam previo al cateterismo cardíaco izquierdo por vía radial reduce la incidencia de espasmo radial y la necesidad de cambio del sitio de acceso arterial, sin asociarse a eventos adversos graves.

\section{ABSTRACT}

Objective. To assess the efficacy and safety of midazolam in the prevention of radial spasm by via transradial cardiac catheterization. Material and methods. Controlled clinical trial, randomized and double blind. 300 patients was randomized (2 groups of 150) to the treatment of midazolam prior to proceeding with the control group (saline ). Results. Radial spasm in 102 patients (34\%) have been documented. In the midazolam Group achieved a significant reduction in the incidence of radial spasm:19 (18.6\%) vs 83 (81.4\%); $p=0.0004 ; R R=0.28(0.49-0.43)$. The need for change of arterial radial access was significantly reduced, $p=0,0049 ; R R=0.61$ (0.17-1.01). Only 3 patients have been documented with drowsiness as an adverse drug event. Conclusions. The use of Midazolam prior to the left heart catheterization by radial artery reduces the incidence of radial spasm and the need for change in the arterial access site, without associated with serious adverse events.

Keywords: radial artery spasm, radial access, midazolam, prevention.

Palabras claves: espasmo de la arteria radial, midazolam, prevención.

Revista Argentina de Cardioangiología Intervencionista 2018;9(1):039-046. Doi: 10.30567/RACI/201801/0039-0046

\section{Cuadro de abreviaturas:}

\begin{tabular}{|l|l|}
\hline ATR & acceso transradial. \\
\hline ICP & intervencionismo coronario percutáneo. \\
\hline ATF & acceso transfemoral. \\
\hline EAM & eventos adversos mayores. \\
\hline EAR & espasmo de la arteria radial. \\
\hline IMC & índice de masa corporal. \\
\hline
\end{tabular}

1. Departamento de Cardiología intervencionista. Hospital Clínico Quirúrgico "Hermanos Ameijeiras". La Habana, Cuba.

2. Departamento de Estadística e Investigación. Hospital Clínico Quirúrgico "Hermanos Ameijeiras". La Habana, Cuba.

$\triangle$ Correspondencia:Dr. Suilbert Rodríguez Blanco. suilbertr@infomed.sld.cu

Los autores no declaran conflictos de intereses.

Recibido: 21/12/2017| Aceptado:04/03/2018

\section{INTRODUCCIÓN}

El acceso transradial (ATR) en la coronariografía diagnóstica y en el intervencionismo coronario percutáneo (ICP) ha emergido hace varios años como la vía preferida por muchos cardiólogos intervencionistas, probablemente por la evidencia que lo asocia a menores complicaciones vasculares, mayor comodidad para el paciente, movilización más temprana, menos estadía hospitalaria ${ }^{1,2}$ y mejores resultados en pacientes de alto riesgo y con síndrome coronario agudo ${ }^{3,4}$, en comparación con otras vías de acceso.

En el Hospital Hermanos Ameijeiras, Cuba, al compararlo con el acceso transfemoral (ATF), Almeida $\mathrm{J}^{5}$ reportó que el ATR se acompañó de menos eventos adversos mayores (EAM) después del proceder y mostró una mayor probabilidad de estar libre de EAM a los 30 días. La tasa de éxito y el tiempo de fluoroscopia fueron 
TABLA 1. Midazolam en la prevención del espasmo de la arteria radial. Variables clínicas de control.

\begin{tabular}{|l|c|c|c|}
\hline Variables & $\begin{array}{c}\text { Grupo I } \\
\text { Midazolam } \\
\mathrm{n}=150(\%)\end{array}$ & $\begin{array}{c}\text { Grupo II } \\
\mathrm{NaCl} \\
\mathrm{n}=150(\%)\end{array}$ & $\begin{array}{c}\text { Valor } \\
\text { de } \mathrm{p}\end{array}$ \\
\hline Edad, años & $60,3 \pm 5,2$ & $59,4 \pm 5,9$ & 0,26 \\
\hline Mujeres & $63(42)$ & $54(36)$ & 0,81 \\
\hline Fumador activo & $54(36)$ & $48(32)$ & 0,52 \\
\hline HTA & $79(52,6)$ & $69(46)$ & 1,04 \\
\hline Diabetes mellitus & $43(28,6)$ & $48(32)$ & 0,67 \\
\hline IMC kg/m ${ }^{2}$ & Media=25,289 & Media=26,476 & 0,16 \\
\hline Diagnóstico clínico & & & \\
\hline AEEC & $82(54,6)$ & $88(58,6)$ & 0,94 \\
\hline SCASEST & $30(20)$ & $34(22,6)$ & 1,01 \\
\hline SCACEST & $6(4)$ & $3(2)$ & 1,00 \\
\hline Prequirúrgicos & $32(21,3)$ & $25(16,6)$ & 0,10 \\
\hline Amplitud de pulso radial & & & \\
\hline Tipo 1 & $35(23,3)$ & $14(9,3)$ & 0,12 \\
\hline Tipo 2 & $68(45,3)$ & $66(44)$ & 0,57 \\
\hline Tipo 3 & $47(31,3)$ & $70(46,6)$ & 0,5 \\
\hline
\end{tabular}

Fuente: base de datos.

similares en ambas vías, pero con un menor costo institucional y una mejor relación costo efectividad para el abordaje transradial.

Uno de los problemas más frecuentes, una vez iniciado el ATR, es la necesidad de cambiar el sitio de acceso arterial, debido a la aparición de un severo espasmo arterial radial que imposibilita el adecuado avance y manipulación de los dispositivos por esta vía ${ }^{1}$. Este cambio de abordaje no solo incrementa la duración del procedimiento y su complejidad, sino que es un importante predictor de complicaciones vasculares mayores en estos pacientes $^{2}$.

El espasmo de la arteria radial (EAR) es definido como la reducción brusca, repentina y temporal de su calibre, diagnosticado clínica y/o angiográficamente, durante el proceder ${ }^{6}$. Clínicamente se presenta con dolor en el antebrazo que aumenta con la movilización de los dispositivos intraarteriales y dificultad en el desplazamiento de estos, puede existir pérdida del pulso radial y caída de la presión en esta arteria. El arteriograma radial confirma el espasmo, confirmación importante (aunque no necesaria) pues en ocasiones el dolor es causado por el desplazamiento de los dispositivos ante factores como: tortuosidad o espiral de la arteria radial, braquial y/o subclavia. El EAR puede tener diferentes grados de seriedad y ocurrir en cualquier etapa del proceder ${ }^{7}$.

El mecanismo exacto que causa el espasmo radial no está del todo esclarecido; se conoce la existencia de factores clínicos, factores relacionados con el proceder y factores anatómicos que predisponen a la aparición del mismo ${ }^{8}$. Como factores clínicos se mencionan: el sexo femenino, edades tempranas, bajo índice de masa corporal (IMC) y diabetes mellitus. Entre los relacionados con el proceder se citan: fallos sucesivos en el intento de canalizar la arteria radial, introducir la guía radial en una ramificación de la arteria, dispositivos intraar-
TABLA 2. Midazolam en la prevención del espasmo de la arteria radial. Variables de control relacionadas con el proceder.

\begin{tabular}{|c|c|c|c|}
\hline Variables & $\begin{array}{c}\text { Grupo I } \\
\text { Midazolam } \\
\mathrm{n}=150(\%)\end{array}$ & $\begin{array}{c}\text { Grupo II } \\
\mathrm{NaCl} \\
\mathrm{n}=150(\%)\end{array}$ & $\begin{array}{l}\text { Valor } \\
\text { de } p\end{array}$ \\
\hline Radial derecha & $133(88,6)$ & $125(83,3)$ & 1,00 \\
\hline \multicolumn{4}{|l|}{ Número de punciones } \\
\hline$\leq 2$ & $106(70,6)$ & $129(86)$ & 0,84 \\
\hline$>2$ & $44(29,4)$ & $21(14)$ & 0,27 \\
\hline Dispositivos hidrofilicos & $148(98,6)$ & $149(99,3)$ & 1,00 \\
\hline \multicolumn{4}{|l|}{ Diámetro del introductor } \\
\hline $5 \mathrm{~F}$ & $81(54)$ & $78(52)$ & 0,87 \\
\hline $6 \mathrm{~F}$ & $69(46)$ & $72(48)$ & 0,51 \\
\hline Tiempo de procedimiento (min) & Media 20,01 & Media 23,28 & 0,06 \\
\hline \multicolumn{4}{|l|}{ Número de catéteres utilizados } \\
\hline$\leq 2$ & $116(77,3)$ & $112(74,6)$ & 1,02 \\
\hline$>2$ & $34(22,6)$ & $38(25,3)$ & 0,94 \\
\hline Volum. de contraste utilizado (ml) & Media 50,15 & Media 55,90 & 0,26 \\
\hline $\begin{array}{l}\text { Anatomía compleja que } \\
\text { favorezca el espasmo }\end{array}$ & $21(14)$ & $11(7,3)$ & 0,11 \\
\hline
\end{tabular}

Fuente: base de datos.

teriales de gran calibre, proceder de larga duración, mayor número de catéteres utilizados y el tiempo de experiencia del operador, ya que la poca experiencia se asocia a mayor probabilidad de espasmo. Como factores anatómicos: pequeño diámetro de la arteria radial, presencia de lesiones ateroscleróticas en esta, tortuosidad y espiral arterial.

La incidencia de espasmo durante la cateterización cardíaca reportada en la literatura oscila en el rango de 4 a $20 \%{ }^{9-11}$. Este rango tan amplio, a nuestro juicio, se debe a la variación en los protocolos de utilización de cóctel antiespasmódico usado por diferentes grupos de trabajo, los criterios utilizados en la definición de EAR, la selección de introductores y catéteres y la curva de aprendizaje de los operadores. Kristic I y colaboradores ${ }^{12}$, en un metaanálisis que incluyó a 7197 pacientes, reportaron una incidencia de $14,7 \%$ de EAR, donde el uso de dispositivos hidrofílicos redujo la incidencia en $1 \%$, mientras que la aplicación de verapamilo y nitroglicerina intraarterial en un $3,8 \%$. Recientemente, en una serie que evaluó las causas de fracaso del ATR13, se encontró una incidencia de $17 \%$ de EAR.

Existen varias medidas orientadas a la prevención y tratamiento del EAR utilizadas durante el proceder, siendo el cóctel antiespasmódico que se emplea por vía intraarterial parte de estas. En una comparación de 3 diferentes métodos de cóctel antiespasmódico 9 (grupo A: heparina, lidocaína, diltiazem y nitroglicerina; grupo B: heparina, lidocaína, diltiazem y nitroprusiato; y grupo C: heparina, lidocaína, diltiazem, nitroglicerina y nitroprusiato) no se encontraron diferencias en la ocurrencia de espasmo radial, Un análisis posterior demostró variables predictoras independientes de espasmo radial, como el diámetro de la arteria radial y la relación diámetro del dispositivo arterial/diámetro de la arteria radial, mientras 
TABLA 3. Midazolam en la prevención del espasmo de la arteria radial. Variables de respuesta. Eficacia del midazolam en la prevención del espasmo radial.

\begin{tabular}{|l|c|c|c|c|c|}
\hline Variables & $\begin{array}{c}\text { Total de pacientes } \\
\mathrm{N}=300(\%)\end{array}$ & $\begin{array}{c}\text { Grupo I } \\
\text { Midazolam } \\
\mathrm{N}=150(\%)\end{array}$ & $\begin{array}{c}\text { Grupo II } \\
\mathrm{NaCl} \\
\mathrm{N}=150(\%)\end{array}$ & Valor de $\mathrm{p}$ & $\begin{array}{c}\text { Riesgo relativo } \\
\mathrm{RR}(\mathrm{IC})\end{array}$ \\
\hline Espasmo Radial & $\begin{array}{c}102(100) \\
\left(34^{*}\right)\end{array}$ & $19(18,6)$ & $83(81,4)$ & 0,0004 & $0,28(0,49-0,43)$ \\
\hline Severidad del espasmo radial & & & & & - \\
\hline Grado 1 & $47(100)$ & $10(21,3)$ & $37(78,7)$ & & $0,53^{* *}$ \\
Grado 2 & $42(100)$ & $8(19,1)$ & $34(80,9)$ & $1,2(0,49-2,57)^{* * *}$ \\
Grado 3 & $13(100)$ & $1(7,7)$ & $12(92,3)$ & $2,77(0,39-19,67)^{* * *}$ \\
\hline Necesidad de cambio de vía de acceso arterial. & $13\left(4,3^{*}\right)$ & $1\left(0,3^{*}\right)$ & $12\left(4^{*}\right)$ & $0.049^{* * * *}$ & $0,61(0,17-1,07)$ \\
\hline
\end{tabular}

Fuente: base de datos. *\% del total de casos estudiados. **Significación estadística de la variable severidad del espasmo radial. ***Tomando como referencia el EAR grado 1. *** Significación estadística en tre la variable necesidad de cambio de vía de acceso v el uso de midazolam.

que Vuurmans et al. ${ }^{14}$, en una revisión, encontraban que la combinación más efectiva para evitar el EAR fue nitroglicerina, verapamilo y heparina. Combinación esta que se utiliza en nuestro laboratorio del Hospital Hermanos Ameijeiras y en muchos en el mundo.

A pesar de estas medidas farmacológicas de prevención del espasmo radial, la incidencia de este reportada mundialmente sigue siendo alta.

Por otro lado, se conoce que el verapamilo puede producir hipotensión y está relativamente contraindicado en los pacientes con disfunción severa del ventrículo izquierdo ${ }^{6}$. La heparina es administrada como parte del cóctel para la prevención de la trombosis de los dispositivos intraarteriales pero puede irritar las arterias de pequeño calibre, su inyección rápida en bolo puede provocar dolor y producir espasmo, por cuya razón en algunos laboratorios se administra por vía intravenosa. Otro método preventivo incluye el uso de dispositivos hidrofílicos, que puede reducir la incidencia de EAR en más de $50 \%{ }^{15}$.

La experiencia del operador influye en la aparición de EAR, dada su relación con los factores de espasmo radial dependientes del proceder. El operador debe tener en cuenta que el tono muscular de la arteria radial es sensible al tono simpático y que el EAR puede ser desencadenado por la ansiedad, el miedo y el dolor. En este contexto, la sedación puede ser efectiva en el alivio de estos desencadenantes, lo que podría reducir la frecuencia de espasmo radial, dando a la sedación un papel importante en el cateterismo cardíaco transradial.

La vasodilatación inducida por benzodiazepinas es un efecto bien documentado. Se ha demostrado que bajas concentraciones de midazolam inducen vasodilatación por un mecanismo dependiente del endotelio, mientras que altas concentraciones inducen vasodilatación independiente del endotelio y sus efectos parecen secundarios a la disminución de la resistencia periférica ${ }^{16}$. Este medicamento provoca hiperpolarización en las células del músculo liso humano, probablemente por activación de canales de potasio, produciendo relajación del músculo y dilatación de vaso $^{17}$, y atenuando la vasoconstricción resultante del estímulo adrenérgico ${ }^{18}$.
TABLA 4. Midazolam en la prevención del espasmo de la arteria radial. Anatomía arterial compleja que favorece el espasmo de la arteria radial.

\begin{tabular}{|l|c|}
\hline Anatomía favorable para espasmo radial & $\mathbf{N}\left(\%^{*}\right)$ \\
\hline Inserción alta de la radial & $9(3)$ \\
\hline Loop de arteria innominada & $6(2)$ \\
\hline Loop arteria braquial & $4(1,3)$ \\
\hline Arteria subclavia lusoria & $3(1)$ \\
\hline Loop arteria subclavia & $3(1)$ \\
\hline Arteria radial hipoplásica & $2(0,6)$ \\
\hline Arteria subclavia tortuosa & $2(0,6)$ \\
\hline Loop arteria radial & $1(0,3)$ \\
\hline Arteria braquial tortuosa & $1(0,3)$ \\
\hline Arteria innominada lusoria & $1(0,3)$ \\
\hline Total & $32(10,6)$ \\
\hline
\end{tabular}

Fuente: base de datos. *Del total de pacientes estudiados.

El efecto sedante de esta droga es particularmente relevante en la sobreactivación adrenérgica en circunstancias de estrés inducido, como los procederes de cateterización cardíaca. En este sentido, un estudio conducido por Suleyman ${ }^{19}$ evaluó la ansiedad como factor de riesgo para desarrollar espasmo radial durante el cateterismo cardíaco y concluyó que la ansiedad se relacionaba significativamente con el EAR $(\mathrm{p}<0,0007)$.

El midazolam se caracteriza por el pronto comienzo de su acción farmacológica y por la brevedad de la misma dada su rápida metabolización. Tiene amplio margen terapéutico toda vez que su toxicidad es baja con un efecto sedante de gran intensidad. Su distribución por vía intravenosa se produce unida a proteínas plasmáticas, con un metabolismo hepático, donde es hidroxilada por la enzima 3A4 del citocromo P450. Una de las indicaciones conocidas de este fármaco es la sedación consciente antes de intervenciones diagnósticas o terapéuticas ${ }^{20,21}$.

Los efectos adversos de este fármaco son muy infrecuentes y se asocian a altas dosis y a una administración rápida. No encontramos reportes de eventos adversos graves asociados al uso de midazolam cuando este se utiliza en el tratamiento del EAR, por lo que consideramos que la relación riesgo-beneficio en el uso de este medicamento está a favor del beneficio potencial que proporcionaría.

Recientemente, se demostró que la combinación de opioides y benzodiazepinas administrados rutinaria- 
TABLA 5. Midazolam en la prevención del espasmo de la arteria radial. Análisis multivariado de variables relacionadas con la presencia de espasmo radial.

\begin{tabular}{|l|c|c|}
\hline Variables & Valor de $\mathrm{p}$ & Hazard ratio: HR (IC) \\
\hline Uso de Midazolam & 0,004 & $0,19(0,071-0,261)$ \\
\hline Sexo femenino & 0,04 & $2,7(1,6-3,2)$ \\
\hline Pulso radial tipo 1 & 0,01 & $3,7(3,0-4,1)$ \\
\hline $\begin{array}{l}\text { Anatomía compleja que } \\
\text { favorece el espasmo radial }\end{array}$ & 0,03 & $2,1(1,09-3,04)$ \\
\hline
\end{tabular}

Fuente: base de datos.

mente durante el proceder transradial se asociaba a una reducción del espasmo de esta arteria ${ }^{22}$; esta serie no estudió las posibles reacciones adversas asociadas al uso de estos medicamentos.

La sedación y analgesia del paciente son usadas en el manejo del espasmo radial durante el proceder ${ }^{23,24}$. En el Hospital Ameijeiras se utiliza midazolam, de 1 a 2 $\mathrm{mg}$, como parte del tratamiento del EAR durante el cateterismo transradial, pero no existe una evidencia sólida que respalde el uso rutinario y efectivo de este medicamento en la prevención del EAR y que permita promover su uso en todos los centros donde se realice cateterismo transradial.

Problema práctico. La incidencia reportada mundialmente del EAR ${ }^{9-11,13}$ llega hasta un 20\%, lo que afecta considerablemente el éxito en la realización del proceder. Se conocen algunos factores predisponentes, pero no la magnitud en que estos influyen en el EAR, que puede ser desencadenado por el miedo y la ansiedad. Conocemos el empleo del midazolam como sedante para tratar el EAR, pero no su eficacia y seguridad en la prevención del mismo.

Problema científico. ¿Es eficaz y seguro el uso de midazolam en la prevención del espasmo radial durante la cateterización cardíaca mediante ATR?

\section{Hipótesis}

En los pacientes en que se utiliza el ATR, la administración de midazolam preproceder a dosis de $2 \mathrm{mg}$, en menores de 70 años y $1 \mathrm{mg}$ en pacientes con 70 años y más, se asocia con una reducción de al menos $40 \%$ en la incidencia de EAR, sin que se produzcan eventos adversos graves al midazolam.

\section{Justificación}

Una vez demostradas las ventaja del ATR , $^{5}$ se impone la sistematización y perfeccionamiento de esta vía, donde la búsqueda de soluciones a problemáticas como el EAR será esencial. En los últimos años han aparecido publicaciones que abordan formas de predicción ${ }^{25}$ y prevención ${ }^{22,26}$ de esta complicación. Con la realización de este ensayo clínico, se pretende encontrar evidencias científicas más sólidas acerca del uso del midazolam preproceder en la prevención del espasmo de la arteria radial y las condiciones que en nuestro medio influyen en dicha complicación.

\section{OBJETIVOS}

Objetivo general. Evaluar la eficacia y seguridad del midazolam en la prevención del espasmo de la arteria radial en los pacientes sometidos a cateterismo cardíaco por vía transradial.

\section{Objetivos específicos}

1. Evaluar la eficacia del midazolam en la aparición e intensidad del espasmo de la arteria radial.

2. Determinar la influencia independiente de algunas variables en la aparición del espasmo de la arteria radial.

3. Identificar los eventos adversos relacionados con la administración del midazolam.

\section{MATERIAL Y MÉTODOS}

Tipo de estudio: ensayo clínico controlado, aleatorizado, a doble ciegas.

Universo: pacientes adultos a los que se les indicó realizar cateterismo cardíaco izquierdo.

\section{Criterios de exclusión:}

- Hipersensibilidad conocida o historia de reacción adversa a las benzodiazepinas y/o midazolam.

- Pacientes en shock cardiogénico.

- Pacientes con enfermedad renal crónica en estado terminal o insuficiencia renal aguda.

- Pacientes en insuficiencia hepática severa.

- Pacientes con Insuficiencia respiratoria aguda.

- Pacientes que se nieguen a participar en el estudio.

\section{Criterios de salida:}

- Pacientes en los cuales no se realice el cateterismo cardíaco luego de administrar el fármaco.

- Pacientes a los cuales no se les canalice la arteria radial.

\section{Tamaño muestral y cálculo:}

Teniendo en cuenta que se reportan cifras de incidencia del EAR entre el 4 y el 20\%, tomamos una cifra base de $15 \%$ para incidencia de EAR en pacientes no tratados. A partir de esta cifra, si se desea detectar una reducción absoluta de $6 \%$, se necesitan 150 pacientes en cada grupo para lograr una potencia de $80 \%$ con un nivel de confianza del 95\%. Esta muestra está conformada por 150 pacientes en cada grupo, a los que se les canalizó la arteria radial con el objetivo de realizar un cateterismo cardíaco izquierdo, desde febrero de 2016 hasta junio de 2016.

Diseño del ensayo: se asignaron de forma aleatoria los pacientes seleccionados a uno de 2 grupos, un grupo de tratamiento (grupo I) y otro control con placebo (grupo II).

Razón de asignación: 1 a 1, que implica grupos de igual tamaño. Estos pacientes no conocieron el grupo al que pertenecen. 
- El grupo de tratamiento (I) está formado por 150 pacientes de forma aleatoria, los cuales recibieron, 10 minutos antes del proceder, la administración de midazolam intravenoso y una vez comenzado el proceder, recibieron el resto de las medidas preventivas de EAR, que incluye el cóctel antiespasmódico.

- El grupo de control (II) está formado por 150 pacientes de forma aleatoria, los cuales recibieron, 10 minutos antes del proceder, la administración de solución salina al 0,9\% intravenosa y una vez comenzado el proceder, recibieron el resto de las medidas preventivas de EAR, que incluye el cóctel antiespasmódico.

Generación de secuencia: la secuencia de aleatorización se generó con el programa Epidat versión 3.1 y se seleccionó la opción de grupos equilibrados. Con esta opción se logró que la asignación sea más balanceada y en todo momento los grupos se mantuvieron con un número similar de pacientes.

Enmascaramiento: dos licenciados en enfermería fueron los encargados de abrir el sobre correspondiente, preparar la medicación y aplicarla, sin revelar al paciente o médico si es del grupo tratado o del grupo control.

\section{Variables de respuesta}

\section{De eficacia:}

- Espasmo radial: basándose en la presencia de dolor en el antebrazo del paciente que aumenta con la movilización del catéter y la imposibilidad de su manipulación.

- Severidad o grado del espasmo radial: grado 1, grado 2 o grado 3. Si el espasmo fue leve con posibilidad de continuar el proceder, sin tratamiento específico para este, se clasificó como grado 1; si el espasmo necesitó tratamiento específico para continuar el proceder por esta vía, se clasificó como grado 2 y si el espasmo impidió continuar con el proceder por esta vía, se clasificó como grado 3, lo que implica cambio de la vía de acceso.

De seguridad: (eventos adversos del midazolam).

\section{Variables de control}

Se incluyeron aquellas variables clínicas y dependientes del proceder que pudieran influir en los resultados del estudio y su generalización.

\section{Consideraciones éticas}

Este ensayo clínico fue conducido de acuerdo con todo lo establecido en la última revisión de la Declaración de Helsinki. Se informó a los pacientes involucrados sobre las características del ensayo y los procedimientos para solicitar y obtener la aprobación de participación de estos y la constancia escrita de esta. El estudio fue aprobado por el Comité de Ética de Investigación Científica y al Consejo Científico del centro.

\section{Técnica y procedimientos}

En caso del grupo de tratamiento, una jeringuilla de $10 \mathrm{ml}$, que contenía midazolam (ampolla de $10 \mathrm{mg}$ en $2 \mathrm{ml}$ ), se diluyó 1 ampolla en $8 \mathrm{ml}$ de solución salina al 0,9\% completando los $10 \mathrm{ml}$, a los pacientes menores de 70 años, se les administró $2 \mathrm{mlL}(2 \mathrm{mg})$ y $1 \mathrm{ml}$ $(1 \mathrm{mg})$ a los pacientes de 70 años o más; si el proceder se prolongaba más de una hora se repite la dosis. En el grupo de control se preparó una jeringuilla de $10 \mathrm{ml}$, que contenía $10 \mathrm{ml}$ de solución salina al 0,9\%, administrándose $2 \mathrm{ml}$ a los pacientes menores de 70 años y 1 $\mathrm{ml}$ a los de 70 o más años; si el proceder se prolongaba más de una hora se repite la dosis.

En ambos grupos, para canalizar la arteria radial se utilizó la técnica de Seldinger ${ }^{27}$ y se aplicó el resto de las medidas antiespasmódicas (incluido el cóctel antiespasmódico: nitroglicerina, verapamilo y heparina).

\section{RESULTADOS Y DISCUSIÓN}

De febrero a junio de 2016 se encontraron inicialmente elegibles para el estudio un total de 313 pacientes consecutivos; de estos, 7 no aceptaron participar en el estudio. Del resto, 306 pacientes, en 6 casos no fue posible la canalización de una arteria radial, por lo que se aleatorizaron 300 pacientes, 150 a cada grupo (Figura 1).

Las Tablas 1 y 2 muestran las características basales de control en cuanto a elementos clínicos y relacionados con el proceder, respectivamente.

No se observan diferencias entre los grupos en cuanto a las variables clínicas (Tabla 1) ni en cuanto a variables dependientes del procedimiento (Tabla 2), evidenciando homogeneidad en ambos grupos incluidas las variables que la literatura mundial recoge como las más relacionadas con el EAR: sexo femenino, el IMC bajo, la baja amplitud de pulso radial, definido en este estudio como pulso tipo 1, el número de punciones para canalizar la arteria, el tiempo total del procedimiento y la presencia de anatomía compleja del eje arterial del miembro superior utilizado que favorezca la aparición de espasmo.

En cuanto al tiempo del procedimiento hubo una tendencia a menos tiempo de procedimiento en el grupo de tratamiento, pero esto puede estar influido por la presencia de mayor frecuencia de EAR en el grupo control, ya que el tiempo prolongado no solo es un factor de riesgo para el EAR sino que este puede prolongarse como consecuencia del espasmo.

De las mujeres, con el 39\% (117 casos), se conoce que presentan una arteria radial de menor calibre que la de los hombres, con una incidencia mayor de test de Allen negativo ${ }^{28}$. El IMC $\left(\mathrm{kg} / \mathrm{m}^{2}\right)$ bajo, el hábito de fumar y la diabetes mellitus, factores de riesgo para el espasmo, estuvieron representados en el 10, 34 y 30,33\%, respectivamente. Esos factores se asocian a disfunción endotelial y mayor inervación adrenérgica, favoreciendo el EAR. El otro factor de riesgo independiente es la am- 


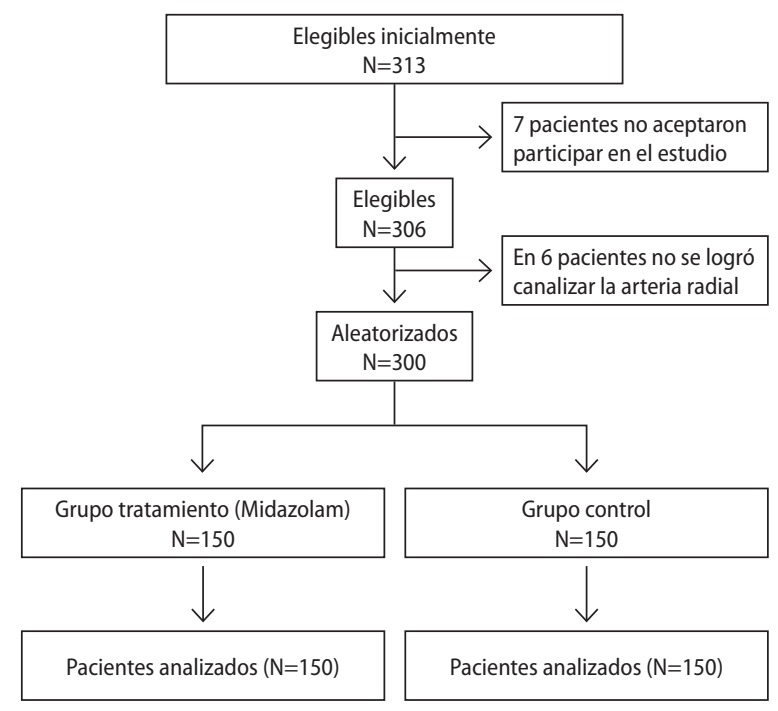

Figura 1. Flujograma del estudio. Pacientes aleatorizados a ambos grupos.

plitud de pulso radial: el pulso tipo 1, de baja amplitud, se encontró en el 16,3\% y otras series lo han relacionado con arterias radiales de menor calibre y mayor dificultad de canulación arterial ${ }^{29}$.

La Tabla 3 y la Figura 2 muestran la eficacia del midazolam en la prevención del espasmo de la arteria radial, logrando una reducción significativa de la presencia de espasmo en el grupo de tratamiento con midazolam $(\mathrm{p}=0,0004)$, además de una reducción en valores absolutos de todas las formas de severidad de este en los tratados previamente con el fármaco, incluyendo la necesidad de cambio de vía de acceso. El análisis univariado de la necesidad de cambio de vía de acceso arterial mostró una reducción significativa en el grupo de tratamiento: $\mathrm{p}=0,049$; $\mathrm{RR}=0,61(0,17-1,07)$.

En el presente estudio, se documentó la presencia de EAR en 102 pacientes para el 34\%, por encima de la incidencia publicada en otras series ${ }^{9-11,13}$. Esto podría tener su explicación en la definición de EAR utilizada en cada investigación (algunas incluyen criterios angiográficos) o a la utilización en nuestro medio de material no específico para el cateterismo cardíaco por vía radial. Sin embargo el espasmo moderado y severo se encontró en 55 pacientes para un 18,33\%, más comparable a los datos internacionales.

En el grupo de midazolam se evidenció espasmo en $19(18,6 \%)$ casos vs. $83(81,4 \%)$ casos del grupo control con placebo, $\mathrm{p}=0.0004 ; \mathrm{RR}=0.28(0,19-0,43)$ con una reducción de 64 casos de espasmo lo que ofrece una protección a los pacientes tratados con midazolam previo al proceder. El efecto sedante y analgésico que se logra con esta dosis de midazolam tiene un beneficio antiespasmódico, esto ha sido poco estudiado y son escasos los reportes del uso de este fármaco en la prevención de EAR, sin embargo basados en esta teoría, Deftereos y colaboradores ${ }^{22}$ encontraron que la administración rutinaria de do- sis bajas de fentanilo y midazolam durante el proceder de ICP se asocia a una reducción significativa de la incidencia de EAR.

Además de la disminución de la incidencia, se encontró una disminución del riesgo de severidad de este, paralelo a un aumento del riesgo en la severidad del espasmo en el grupo control. Tomando como referencia el espasmo grado 1, la relación de este con el grado 2 es $R R=1,2(0,49-2,57)$ y la relación con el grado 3 es $R R=2,27$ (0,39-19,67), siendo el espasmo moderado y severo menor en el grupo de tratamiento, $9 v$ s. 46 casos. Dentro de las complicaciones del espasmo radial se encuentra la necesidad de cambio de vía de acceso. Esta, además de aumentar el tiempo del procedimiento, incrementa el riesgo de sangrado, el dolor y la disconformidad del paciente. En esta investigación esa complicación se redujo significativamente con el uso de midazolam previo al proceder. A nuestro criterio, una variable de mucho peso en la evaluación del perfeccionamiento de la técnica del cateterismo transradial.

En el estudio PRESTO ACS (Comparison of Early Invasive and Conservative Treatment in Patients With Non-ST-Elevation Acute Coronary Syndromes) ${ }^{30}$ la vía radial estuvo asociada con una reducción significativa de las complicaciones hemorrágicas comparadas con la vía femoral, este beneficio se perdía cuando una vez iniciado el acceso transradial había necesidad de cambio de vía; y en otra serie ${ }^{23}$, donde se utilizó midazolam en el tratamiento del EAR, se logró concluir el proceder por esta vía en un grupo considerable de pacientes.

La Tabla 4 muestra la presencia de alteraciones anatómicas del eje radial-braquial-axilar-subclavio y del tronco braquiocefálico de nuestra serie,con 32 casos, donde la más representada fue el origen alto de la radial (9 pacientes), comparado con lo reportado mundialmente; en estos la manipulación de los dispositivos para vencer estas dificultades anatómicas favorece la aparición del espasmo.

El análisis multivariado (Figura 3 y Tabla 5) muestra que las variables más relacionadas con la aparición de espasmo fueron el pulso radial tipo 1, el sexo femenino y la presencia de anatomía compleja del eje arterial favorecedor de espasmo radial. En este análisis, el uso de midazolam previo al proceder se asoció con menos incidencia de espasmo radial (hazard ratio: 0.19; IC95\%: 0,071-0,261; $p=0,0004)$, con un efecto protector. Donde se necesitó tratar a 3 pacientes para evi$\operatorname{tar} 1$ EAR.

La asociación entre pulso radial débil, tortuosidad de la arteria subclavia como anatomía compleja y la presencia de espasmo ha sido documentada en otras publicaciones $^{31}$, donde se demostraron arterias radiales de menor grosor.

En lo relacionado al sexo, Dehghani y colaboradores ${ }^{29}$, en su estudio de pacientes intervenidos por vía radial donde el $17 \%$ fueron mujeres, demuestran que este género se asocia a mayor fallo del procedimiento. 


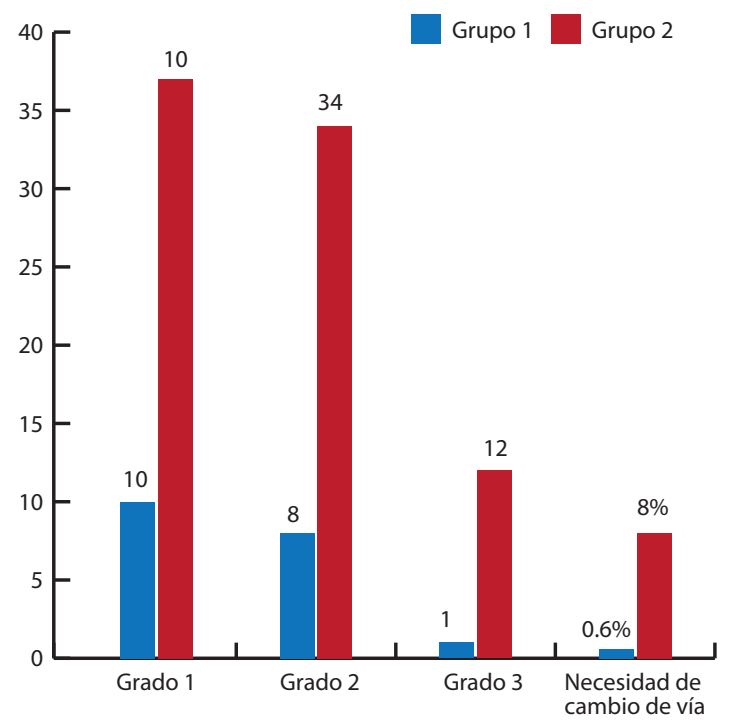

Figura 2. Midazolam en la prevención del espasmo de la arteria radial. Variables de respuesta. Eficacia del midazolam en la prevención del espasmo radial. Fuente: base de datos. *Según total de pacientes en cada grupo.

En el análisis de las variables de seguridad relacionadas con los eventos adversos del midazolam, solo se encontró que 3 pacientes presentaron somnolencia, un $2 \%$ del grupo de tratamiento. La somnolencia constituye un evento adverso menor que en esta serie no necesitó de tratamiento específico para su restablecimiento; este efecto había cesado completamente antes de que el paciente abandonara el salón de hemodinámica. Siendo el uso de este fármaco seguro cuando se utiliza en bajas dosis, administración lenta y en pacientes seleccionados.

\section{BIBLIOGRAFÍA:}

1. Jolly SS, Amlani S, Hamon M, et al. Radial versus femoral access for coronary angiography or intervention and the impact on major bleeding and ischemic events: a systematic review and meta-analysis of randomized trials. Am Heart J. 2009;157:132- 40.

2. Hetherington $S L$, Adam Z, Morley R. Primary percutaneous coronary intervention for acute ST-segment elevation myocardial infarction: changing patterns of vascular access, radial versus femoral artery. Heart. 2009;95:1612-8.

3. Jolly SS,Jolly SS, Yusuf S, et al. Radial versus femoral access for coronary angiography and intervention in patients with acute coronary syndromes (RIVAL): a randomised, parallel group, multicentre trial. Lancet. 2011:377:1409-20.

4. RaoSV, Cohen MG, KandzariDE, etal. The transradial approach to percutaneous coronary intervention: historical perspective, current concepts, and future directions. J Am Coll Cardiol. 2010;55:2187-95.

5. Almeida Gómez J. Evaluación clínica y económica de los abordajes radial y femoral en el cateterismo cardíaco. [Tesis para aspirar al grado de Doctor en Ciencias Médicas]. La Habana. Universidad Médica de la Habana. 2012. Disponible en: http://tesis.repo.sld.cu/579/1/AlmeidaGomez.pdf

6. Ho HH, Jafary FH, Ong PJ. Radial artery spasm during transradial cardiac catheterization and percutaneous coronary intervention: incidence, predisposing factors, prevention, and management. CardiovascRevasc Med. 2012;13(3):193-5

7. Kiemeneij F, Vajifdar BU, Eccleshall SC, et al. Measurement of radial artery spasm using an automatic pullback device. Catheter Cardiovasclnterv. 2001;54(4):437-41.

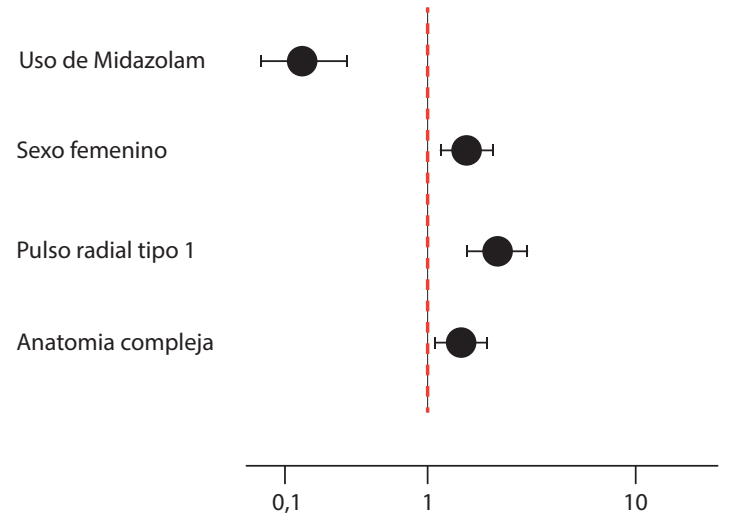

Figura 3. Midazolam en la prevención del espasmo de la arteria radial. Análisis multivariado de variables relacionadas con la presencia de espasmo radial. Fuente: base de datos.

\section{CONCLUSIONES:}

1. En el grupo tratado con midazolam, disminuyó significativamente la frecuencia del espasmo de la arteria radial y la necesidad de cambio de vía de acceso arterial. El uso del midazolam previo al procedimiento se asoció de forma independiente a la prevención del espasmo de la arteria radial.

2. El pulso radial tipo 1, el sexo femenino y la presencia de anatomía arterial compleja se relacionaron de forma independiente con la presencia de espasmo de la arteria radial.

3. El uso de midazolam no se asoció con la aparición de eventos adversos graves.

8. Jia DA, Zhou YJ, Shi DM, et al. Incidence and predictors of radial artery spasm during transradial coronary angiography and intervention. Chin Med J. 2010;123(7):843-7.

9. Coppola J, Patel T, Kwan T, et al. Nitroglycerin, nitroprusside, or both, in preventing radial artery spasm during transradial artery catheterization. J/nvasive Cardiol. 2006;18(4):155-8.

10. Chen CW, Lin CL, Lin TK, et al. A simple and effective regimen for prevention of radial artery spasm during coronary catheterization. Cardiology. 2006; 105(1):43-7.

11. Varenne O, Jegou A, Cohen R, et al. Prevention of arterial spasm during percutaneous coronary interventions through radial artery: the SPASM study. Catheter CardiovascInterv. 2006;68(2):231-5.

12. Kristic I, Lukenda J. Radial artery spasm during transradial coronary procedures. J Invasive Cardiol. 2011;23(12):527-31.

13. Abdelaal E, Brousseau-Provencher C, Montminy S, et al. RiskScore, Causes, and Clinical Impact of Failure of Transradial Approach for Percutaneous Coronary Interventions. J Am CollCardiollntv. 2013;6:1129-37.

14. Vuurmans T, Hilton D. Brewing the right cocktail for radial intervention. Indian Heart J. 2010;62(3):221-5.

15. Rathore S, Stables RH, Pauriah M, et al. Impact of length and hydrophilic coating of the introducer sheath on radial artery spasm during transradial coronary intervention: a randomized study. JACC CardiovascInterv. 2010;3(5):475-83.

16. Colussi GL, Di Fabio A, Catena C, Chiuch A, Sechi LA. Involvement of endothelium-dependent and-independent mechanisms in midazolam-induced vasodilation. Hypertens Res. 2011;34:929-34. 
17. Klockgether-Radke AP, Pawlowski P, Neumann P, et al. Mechanisms involved in the relaxing effect of midazolam on coronary arteries. Eur J Anaesthesiol. 2005:22:135-9.

18. Borges AA, Gomes OM. Effects of midazolam on the contraction and relaxation of segments of thoracic aorta stripped of endothelium and stimulated by adrenaline - experimental study in rabbits. Mol Cell Biochem. 2003:246:13-7.

19. Süleyman E, Ahmet U, Gokhan A, et al. Anxiety Score As a Risk Factor for Radial Artery Vasospasm During Radial Interventions: A Pilot Study. JACC. 2013;62(18 Supp/ C):S1-S2.

20. Hobbs WR, Rall TW, Verdoorm TA. Hipnóticos y sedantes; etanol. En: Marchall BE, Longnecker DE, editors. Las bases farmacológicas de la terapéutica. Mc Graw-Hill Interamericana; 1996. p. 385-421.

21. Formulario nacional de medicamentos [infomed]. Midazolam. [actualizado 07 de septiembre de 2011; citado 12 de agosto 2015]. [aprox. 2 pantallas]. Disponible en: http://fnmedicamentos.sld.cu/index.php? $P=-$ FullRecord\&ID=102

22. Deftereos S, Giannopoulos G, Raisakis K, et al. Moderate Procedural Sedation and Opioid Analgesia During Transradial Coronary Interventions to Prevent Spasm. A Prospective Randomized Study. JACC. 2013;6(3):267-6.

23. Eltahawy E, Cooper Ch. Managing Radial Access Vascular Complications: Recognizing complications associated with transradial access and available management options.[Internet]. Transradialworld; 2010 [cited 2013 dic 23]. Available from: http://Www.transradialworld.com/index.php?pid=1
24. Moscoso N, Sepulveda J, Salas O, et al. Técnicas de acceso transradial en el intervención percutánea coronaria. MedicinaUniversitaria. 2013;15(58):33-39.

25. Giannopoulos G, Kossyvakis C, Driva M, et al. Radial artery flow-mediated dilation predicts arterial spasm during transradial coronary interventions. Catheter Cardiovascinterv. 2011;77(5):649-54.

26. Shroff A, Siddiqui S, Burg A, et al. Identification and management of complications of transradial procedures. CurrCardiolRep. 2013;15(4):350

27. Andrél, Hunghes C, Fajadet J. Radial approach for percutaneous coronary intervention. Euro Intervention. 2009;5:1

28. Kamienski, R.W. and Barnes R.W: Critique of the Allen test for continuity of the palmar arm assessedby Doppler ultrasound. SurgGynecol Obstet.1977;142:861.

29. DehghaniP,MohammadA, BajajR, et al. Mechanism and Predictors of Failed Transradial Approach for Percutaneous Coronary Interventions. JACC Cardiovascular Interventions. 2009; 2(11):1057-64.

30. Sciahbasi A, Pristipino C, Ambrosio G. Arterial access-site related outcomes of patients undergoing invasive coronary procedures for acute coronary syndromes (from the ComPaRison of Early Invasive and Conservative Treatment in Patients With Non-ST-ElevatiOn Acute Coronary Syndromes [PRESTO-ACS] Vascular Substudy). Am J Cardiol 2009;103:796-800.

31. Cha KS, Kim MH, Kim HJ. Prevalence and clinical predictors of severe tortuosity of right subclavian artery in patients undergoing transradial coronary angiography. Am J Cardiol. 2003;92:1220-2. 